\title{
Cavitated Lesion in a Patient Infected by the Human Immunodeficiency Virus (HIV)
}

\author{
Aceituno Cano Alexandra Maria, ${ }^{1, *}$, Gaquez Aguilera Elena ${ }^{1, \text { * }, ~ G a l i n d o ~ F l o r e s ~ M a r i a ~ F e r n a n d a ~}{ }^{2, *}$, \\ Vogt Sanchez Esteban Alessandro, ${ }^{1, *}$, Collado Romacho Antonio Ramon ${ }^{1, *}$ \\ ${ }^{1}$ Department of Internal Medicine, Torrecardenas University Hospital, Almeria, Spain \\ ${ }^{2}$ Department of Orthopedic Surgery and Traumatology, Torrecardenas University Hospital, Almeria, Spain
}

Email address:

alexac265@gmail.com (A. C. A. Maria), elena_gaz_agui@hotmail.com (G. A. Elena), mfernandagalindo@gmail.com (G. F. M. Fernanda), stephgolfista@gmail.com (V.S.E. Alessandro), arcollad@icloud.com (C. R. A. Ramon)

${ }^{*}$ Corresponding author

\section{To cite this article:}

Aceituno Cano Alexandra Maria, Gaquez Aguilera Elena, Galindo Flores Maria Fernanda, Vogt Sanchez Esteban Alessandro, Collado Romacho Antonio Ramon. Cavitated Lesion in a Patient Infected by the Human Immunodeficiency Virus (HIV). International Journal of Biomedical Science and Engineering. Vol. 7, No. 3, 2019, pp. 75-78. doi: 10.11648/j.ijbse.20190703.13

Received: August 8, 2019; Accepted: September 5, 2019; Published: October 2, 2019

\begin{abstract}
It is known that the progressive deterioration of the immune system in the human immunodeficiency syndrome (AIDS), either cellular or humoral level and the obligated role of exchange of the respiratory system with the external environment, makes the incidence of inflammatory, tumoral, and infectious lung processes high in patients infected with the human immunodeficiency virus (HIV). There are several microorganisms that can cause cavitated lung lesions in a HIV patient as Pneumocystis carinii, Mycobacterium tuberculosis or Staphylococcus aureus, among others. Rhodoccoccus equi (R. Equi) is, predominantly, an opportunistic pathogen who can also cause disease in humans, especially in immunocompromised patients. This report describe a case of Rhodococcus equi lung infection in a HIV patient, which highlights the importance of the therapheutic adherence and the epidemiological enviroment in this kind of patients.
\end{abstract}

Keywords: Cavitated Pneumonia, AIDS, Rhodococcus Equi

\section{Introduction}

Rhodococcus equi is an aerobic, strict and intracellular gram-positive coccobacilli, previously classified as Corynebacterium [1], which is a frequent cause of disease in a number of animals, being the most common cause of pneumonia in foals [2]. There is increasing recognition that this pathogen can cause disease in humans, both immunocompetent or immunocompromised individuals, it became more widely recognized as a cause of disease in humans with the onset of the HIV/AIDS epidemic in the 1980s [3]. In more recent years, infections have also been increasingly recognized in patients who have received organ transplants [4]. Lung crhonic infection is the most common pathology caused by this microorganism, but it can also cause isolated bacteriemia, abscesses in any organ and wound infection [5]. Treatment is not well established, antibiotic choices have often included combination therapy with vancomycin, macrolides, fluoroquinolones, rifampin, and imipenem-cilastatin. [6] The duration and intensity of treatment should be determined by the nature of the lesion and the immunologic status of the host. This report describes a case of a 42 years old patient, HIV without therapeutic compliance, with a lung infection due to R. Equi.

\section{Method}

\subsection{History}

This report describes a 42-year-old male with infection with the human immunodeficiency virus type 1 (HIV-1) category C3. Currently in antiretroviral treatment (HAART) with Elvitegravir/cobicistat/Emtricitabina/Tenofovir alafenamida, with poor therapeutic compliance. Native of the Czech Republic, who has lived in Spain (Aguamarga, Almería) continuously for the last 15 years. He works as a carer of horses. Smoker of 11 packages/year and drinker of 
$40 \mathrm{~g}$ alcohol/day. No known drug allergies.

He went to the emergency department with progressive onset fever, up to $39^{\circ} \mathrm{C}$, of one month of evolution, associating dyspnea with moderate efforts and pleuritic features chest pain with brownish expectoration and bloody traces.

\subsection{Physical Examination}

Physical examination revealed a temperature of $38.2^{\circ} \mathrm{C}$, TA $90 / 50 \mathrm{mmHg}$, SatO2 $92 \%$ ambient air. Neat feeling of illness, well perfused and hydrated. No adenopathies were palpable. Oropharynx with lingual leukoplakia. Eupneic at rest, without requiring supplementary oxygen therapy. Conscious, oriented, with normal language and cranial nerves, there were no meningeal signs, muscle balance and normal tendon reflexes. Rhythmic tones without murmurs, vesicular murmur preserved without added noises. Soft abdomen, without masses, visceromegalias or signs of peritoneal irritation. Bilateral symmetrical pulses without edema.

\subsection{Radiological and Laboratory Examinations}

In the complementary tests performed in the emergency room, hemoglobin was $13.6 \mathrm{~g} / \mathrm{dl}$, hematocrit $37.5 \%$ total leukocytes 9160/microL, (neutrophils 91\%, lymphocytes $2.48 \%$ ), total lymphocytes 230/microL. Platelets $316300 /$ microL. The biochemical study was normal, except for $\mathrm{C}$ reactive protein $(\mathrm{PCR}$ ) of $6.26 \mathrm{mg} / \mathrm{dL}$ (normal up to $0.5 \mathrm{mg} / \mathrm{dL}$ ). Urinary sediment without alterations. The chest radiography showed a condensing and cavitated lesion in the right upper lobe (Figure 1). The electrocardiogram showed a sinus rhythm at 98 beats per minute without alterations in repolarization. Given the complementary tests the patient is diagnosed with cavitated pneumonia, with a score of 42 on the FINE scale. He entered in the Internal Medicine plant, in respiratory isolation.

A large microbiological study was carried out and a empirical antibiotic treatment was started with Ceftriaxone $2 \mathrm{~g}$ every 24 hours and Clindamycin $600 \mathrm{mg}$ every 8 hours. Among the results of the study, pneumococcal and legionella antigenuria resulted negative, as well as the serology for brucella or Toxoplasma gondii, hepatitis A, B and C viruses and siphylis. Immunofluorescence for Pneumocystis jiroveci negative. Thoraco-abdominal CT was performed, a lesion compatible with abduced pneumonia was found in LSD, concordant with the image visualized on the previous chest radiograph. The immuno-virological study showed: 217 total lymphocytes, which suppose $13 \mathrm{CD} 4$ lymphocytes $(6 \%)$ and 80 CD8 lymphocytes, with an HIV viral load of $113,526 \mathrm{copies} / \mathrm{ml}$. Given the state of immunosuppression, prophylaxis was initiated with Trimetroprim $160 \mathrm{mg} /$ Sulfamethoxazole $800 \mathrm{mg}$ three times a week.

\section{Result}

After five days of hospitalization, the result of the suptum culture was obtained, Rhodococcus equi and negative serial bacilosocopies were isolated. The antimicrobial treatment was modified, directed according to the antibiogram, with Imipenem 500mg every 6 hours, Clarithromycin 500mg every 12 hours and Rifampicin 600mg a day. After a week of antibiotic treatment, the antiretroviral treatment, that the patient had been voluntarily suspended during the previous 7 months, was reinitiated with Emtricitabine, Tenofovir disoproxil fumarate and Raltegravir.

After three weeks of parenteral treatment, the patient shows significant clinical improvement, so we proceed to hospital discharge with Rifampicin and Clarithromycin until the next review in consultation. After six months of treatment, the patient presents clinical and radiological improvement, remaninig residual lesions in the chest radiography yet.

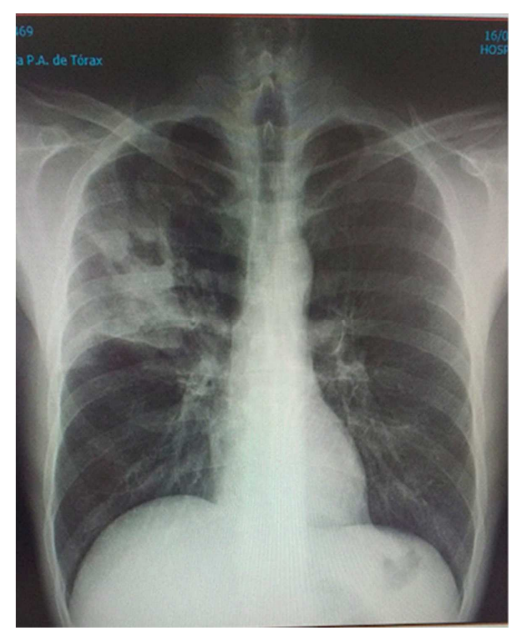

Figure 1. Chest radiography: Cavitated lesion in rhe right upper lobe.

\section{Discussion}

The genus Rhodococcus is composed of several ubiquitous environmental species. These are aerobic, strict and intracellular gram-positive coccobacilli that affect innate immunity, being cellular immunodepression (HIV), hematologic neoplasia, transplantation, and steroid treatment, among others, the risk factors to suffer from this pathogen. The epidemiological environment surrounding the patient is important to establish a diagnostic suspicion, since these microorganisms are usually present in the intestine of herbivorous animals and disseminated in the environment.

Rhodococcus equi, is the most frequent species and has the highest pathogenic capacity in humans, either in immunocompromised people or, less frequently, in immunocompetent ones. It is transmitted by the inhalation of soil particles, by direct ingestion, by contact with a solution of continuity in a mucous membrane or by inoculation in a wound. The main form of transmission is by exposure to soil contaminated by herbivore manure. The most common form of presentation, in immunocompromised patients, is cavitated pneumonia in the upper lobes, while in immunocompetent the extrapulmonary location predominates, affecting the 
central nervous system and the skin [7].

The first case in a patient with HIV infection, was reported in 1986. Since then, there has been a progressive increase in the incidence of infection within this subgroup of the population. It has an inversely proportional relationship to the CD4 lymphocyte count, in fact, in the majority of cases reported, the incidence is higher among those HIV patients who have already suffered opportunistic AIDS-defining diseases [8].

The main affectation of Rhodococcus equi is pulmonary, followed by the presence of nonspecific constitutional syndrome [9]. Clinical symptoms such as fever, pleuritic pain, present an insidious course, which implies a delay in diagnosis and a worse response to antimicrobial treatment [10-11]. The form of presentation at the radiographic level is varied, interstitial, nodular or alveolar infiltrates can be observed. Cavitations can occur in more than $50 \%$ of cases [12]. Extrapulmonary metastases are possible and frequent. The diagnosis is based on the culture of samples of affected tissue in an aerobic environment at a temperature of $37^{\circ} \mathrm{C}$, which ensures high profitability.

Regarding the therapeutic attitude towards Rhodoccocus equi, there is no established regimen. The infection of any location in the immunocompetent patient, except for the central nervous system (CNS), can be treated with a macrolide and rifampin or a quinolone for at least 2 months, orally. The infection in the immunocompromised patient or the CNS infection, in any type of patient, or the serious infection, must be treated with the association of three drugs: vancomycin or carbapenem plus rifampin plus moxifloxacin or macrolide or aminoglycoside, for at least 6 months. Patients with chronic immunosuppression should maintain a chronic suppressive treatment, due to the risk of recurrence [13]. It is not clearly defined with which drugs, although a regimen in oral monotherapy with in vitro activity against Rhodococcus could be sufficient: azithromycin or trimeptrprim/sulfamethoxazole. Quinolones may also be an option. Linezolid is also active in case of allergy.

There are fewer reported cases of $\mathrm{R}$. equi infection in immunocompetent patients who have often responded to short courses of treatment with single antibiotics, although treatment failures still occur [14].

Regarding primary prevention in patients HIV, the most effective measure is the early initiation of HAART. In untreated HIV-infected patients the relative risk of death from R. equi infection was 53 times greater than in those who received appropriate antiretroviral therapy [15].

A possible complication could be the syndrome of immune reconstitution (SIRS), but both, the prognosis and the clinical evolution of the patient, have been favored with the early initiation of HAART.

\section{Conclusion}

The infection by $\mathrm{R}$. equi is an infrequent complication in patients with AIDS, which should be suspected taking into account the epidemiological environment of the patient.
Early diagnosis, combined and prolonged antimicrobial treatment and the initiation of HAART early, can improve the evolution and prognosis of these patients. The immunologic status of the host is, probably, the main determinant of the success of therapy.

\section{Acknowledgements}

No external funding was used in the process of this report. A. C. A. M. drafted the manuscript. A. G. E. and G. F. M. F edited the manuscript. V. S. E. A and C. R. A. R. revised the bibliography. All authors report no conflicts of interest relevant to this article.

\section{References}

[1] Goodfellow M. The taxonomic status of Rhodococcus equi. Vet Microbiol. 1987; 14 (3): 205-9.

[2] Cohen ND. Rhodococcus equi foal pneumonia. Vet Clin North Am Equine Pract. 2014; 30 (3): 609-622.

[3] Arlotti M, Zoboli G, Moscatelli GL, Magnani G, Maserati R, Borghi V, et al. Rhodococcus equi infection in HIV-positive subjects: a retrospective analysis of 24 cases. Scand J Infect Dis. 1996; 28 (5): 463-7.

[4] Arya B, Hussian S, Hariharan S. Rhodococcus equi pneumonia in a renal transplant patient: a case report and review of literature. Clin Transplant. 2004; 18 (6): 748-52.

[5] Kedlaya I., Ing M. B., Wong S. S. Rhodococcus equi infections in immunocompetent hosts: case report and review. Clin Infect Dis. 2001; 32 (3): E39-E46.

[6] Prescott JF. Rhodococcus equi: an animal and human pathogen. Clin Microbiol Rev. 1991; 4 (1): 20-34.

[7] Weinstock D. M., Brown A. E. Rhodococcus equi: an emerging pathogen. Clin Infect Dis. 2002; 34 (10): 1379-1385

[8] Samies JH, Hathaway BN, Echols RM, Veazey JM, Pilón VA. Lung abscess due to Corynebacterium equi. Report of the first case in a patient with acquired inmune deficiency syndrome. Am JMed 1986; 80: 685-8.

[9] Kwa AL, Tam UH, Ryback MJ. Rhodococcus equi pneumonia in patient with inmunodeficiency virus: Case report and review. Pharmacotherapy. 2001; 21: 1998-1002.

[10] Torres M, Arrizalbaga J, Villanueva JL, Gálvez J, Leyes M, Valencia ME, et al. Prognosis and clinical evaluation of infection causes y Rhodococcus equi in HIV infectes patient. A multicenter study of 67 cases. Chest. 2003; 123: 1970-6.

[11] McNeil MM, Brown J. Distribution and antimicrobial susceptibility of Rhodococcus equi from clinical specimens. Eur J. Epidemiol 1992; 8: 437-43.

[12] Verville TD, Huycke MM, Greenfield RA, Fine DP, Kuhls TL, Slater LN. Rhodococcus equi infections of humans. 12 cases and a review of the literature. Medicine (Baltimore). 1994; 73: 119-32.

[13] Menon V, Gottlieb T, Gallaher M, Cheong EL. Persistent Rhodococcus equi infection in a renal transplant patient: case report and review of the literature. Transpl Infect Dis 2012; 14: E126. 
[14] Ng S, King CS, Hang J, Clifford R, Lesho EP, Kuschner RA, et al. Severe cavitary pneumonia caused by a non-equi Rhodococcus species in an immunocompetent patient. Respir Care. 2013; 58 (4): e47-50.
[15] Topino S, Galati V, Grilli E, Petrosillo N. Rhodococcus equi infection in HIV-infected individuals: case reports and review of the literature. AIDS Patient Care STDS. 2010; 24 (4): 21122. 